\title{
Genetic analysis and clinical investigation of a pedigree with multiple endocrine neoplasia type 2A: A case report
}

\author{
HONG-JIN ZOU, YU-SHU LI and ZHONG-YAN SHAN \\ Department of Endocrinology and Metabolism, Institute of Endocrinology, \\ Liaoning Provincial Key Laboratory of Endocrine Diseases, \\ The First Affiliated Hospital of China Medical University, Shenyang, Liaoning 110001, P.R. China
}

Received March 10, 2015; Accepted January 8, 2016

DOI: $10.3892 / \mathrm{ol} .2016 .5028$

\begin{abstract}
Multiple endocrine neoplasia 2A (MEN2A) is characterized by the coexistence of tumors that involve two or more endocrine glands within the same patient, and is defined as the occurrence of medullary thyroid carcinoma in association with pheochromocytoma (PHEO) and parathyroid tumors or hyperparathyroidism. The pathogenesis of MEN2A is due to the mutation of a tyrosine kinase receptor that is encoded by the rearrangement during transfection (RET) proto-oncogene. The mutation often occurs in exon 10q11.2. The present study reports the case of a 73-year-old man with severe hypercalcemia, bilateral adrenal PHEO and a thyroid nodule. A genetic panel was obtained, and the RET mutation was indicated. The pedigree of the patient was also studied. Genetic testing of the patient's son indicated heterozygosity for the same mutation at codon 634. The first symptom of the two patients was PHEO, which is uncommon. In addition, varied phenotypes were identified in the two patients. In the present study, the association between the phenotypic variation of the RET gene and the occurrence of MEN2A is discussed.
\end{abstract}

\section{Introduction}

In 1968, Steiner et al first used the term MEN2, which is now known as multiple endocrine neoplasia 2A (MEN2A) (1). In 1986, Farndon et al decribed familial medullary thyroid carcinoma (MTC), which appears to be closely associated with MEN2A (2). The phenotypes of MEN2A include bilateral multiple MTC, unilateral or bilateral phaeochromocytoma (PHEO), and parathyroid adenoma or hyperplasia (3). In the majority of cases, the diagnosis of PHEO is determined following the diagnosis

Correspondence to: Professor Yu-Shu Li, Department of Endocrinology and Metabolism, Institute of Endocrinology, Liaoning Provincial Key Laboratory of Endocrine Diseases, The First Affiliated Hospital of China Medical University, 155 Nanjing Bei Street, Shenyang, Liaoning 110001, P.R. China

E-mail: liyushu@hotmail.com

Key words: multiple endocrine neoplasia 2A, pheochromocytoma, medullary thyroid carcinoma, parathyroid tumors, phenotype of MTC. The first symptom of the patient in the present study was bilateral PHEO, with a phenotype of parathyroid adenoma. However, the manifestations of MEN2A indicated in the patient's son were of MTC and PHEO, despite the same genotype being present. However, no occurrence of additional mutations was present. These findings inferred that there may be another proto-oncogene dysfunction or activation present, in addition to the multiple superimposed effects of mutation epigenetics. The interaction between these genetic factors and environmental effects may have lead to the varied clinical manifestations.

\section{Case report}

A 73-year-old man presented to The First Affiliated Hospital of China Medical University (Shenyang, China) in july 2013 with persistently increased levels of serum calcium. The patient had undergone a bilateral adrenalectomy for PHEO 4 years previously. An ultrasound examination of the thyroid gland revealed a low echo, 16.0x10.4-mm nodule, in addition to the elevated levels of parathyroid hormone $(81.06 \mathrm{pmol} / \mathrm{l}$; normal range, 0.66-12 pmol/1), serum calcitonin (53.79 pmo/l; normal range, $0.58-22 \mathrm{pmol} / \mathrm{l})$ and serum carcinoembryonic antigen (CEA; $7.24 \mathrm{ng} / \mathrm{ml}$; normal range, 0.0-4.3 ng/ml) indicated by a laboratory examination. A bilateral adrenal ultrasound revealed kidney stones in the left kidney and a contrast-enhanced cervical computed tomography (CT) scan showed a well-defined enhancing lesion in the right thyroid and a lesion in the parathyroid, which suggested the diagnosis of thyroid and parathyroid adenomas. The genomic DNA of the proband was extracted from peripheral blood using a DNA extraction and purification kit (Qiagen $\mathrm{GmbH}$, Hilden, Germany). Shenzhen Huada Gene Technology Co., Ltd., (Shenzhen, China) was authorized to amplify all exons of the rearrangement during transfection (RET) and menin genes, then perform bidirectional sequencing. The heterozygous polymorphism, TGC $\rightarrow$ CGC, at codon 634 (C634R) on the exon 11 rs377767437 sequence of the RET proto-oncogene was detected in the patient, which resulted from the C634R cysteine to arginine substitution. The patient underwent a parathyroid adenoma resection in June 2014 and the diagnosis of parathyroid adenoma was confirmed by histological examination (Fig. 1A), however, the patient refused treatment for the thyroid lesion. The patient was lost to follow-up. 

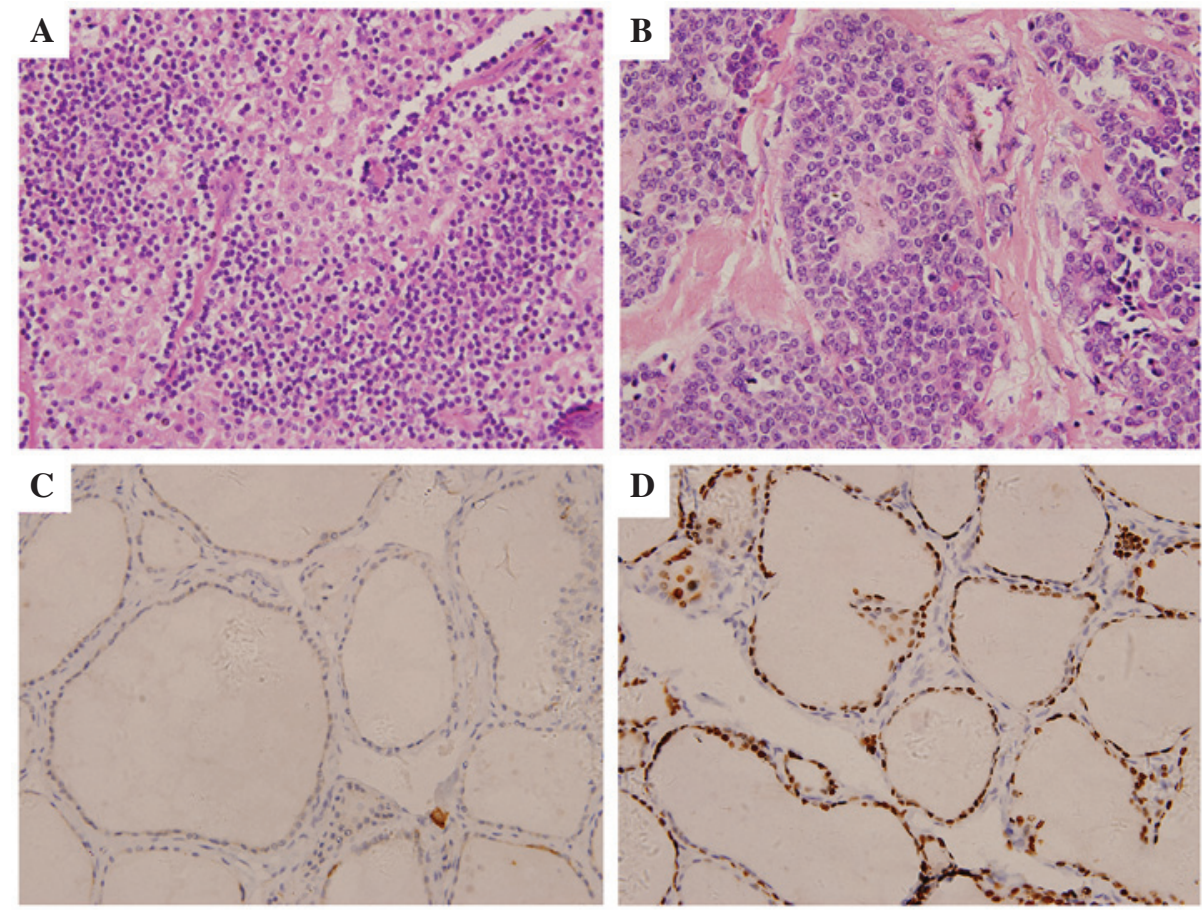

Figure 1. (A) Histopathological findings of the patient's parathyroid adenoma stained with hematoxylin and eosin (magnification, $\mathrm{x} 400$ ), consisting mainly of chief cells, clear cells and oxyphil cells. (B) The post-operative pathology of surgical material from the patient's son, stained with hematoxylin and eosin (magnification, $\mathrm{x} 400$ ), composed of polygonal or plump spindle cells. Characteristic slender fibrovascular septas are present in the carcinoma mass. Island- or rosette-like cell clusters and stromal amyloid deposition are also present. Immunohistochemical anaylsis revealed medullary carcinoma, with positive expression of (C) calcitonin and (D) thyroid transcription factor-1 (magnification, $\mathrm{x} 400$ ).

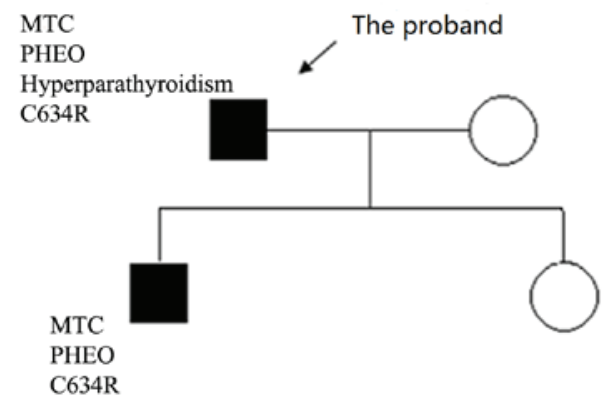

Figure 2. Pedigree of the family. Squares and circles indicate males and females, respectively. MTC, medullary thyroid carcinoma; PHEO, pheochromocytoma; C634R, cysteine-634-arginine substitution.

The 43-year-old son of the patient underwent genetic testing, which indicated heterozygosity for the same mutation at C634R. The symptoms of the son included bilateral PHEO, calcitonin levels of $>1,450 \mathrm{pmol} / 1$, elevated CEA levels $43.6 \mathrm{ng} / \mathrm{ml}$ and normal parathyroid hormone levels. Repeated 24-h urinary tests showed elevated levels of catecholamines, at $1,530.2 \mathrm{~mol} / 24 \mathrm{~h}$ (normal range, 59.1-266 mol/24 h). MTC was diagnosed from the thyroid ultrasound, the elevated calcitonin and CEA levels, and the post-operative pathology (Fig. 1B-D). The post-operative pathology of the patient's son was composed of polygonal or plump spindle cells. Characteristic slender fibrovascular septas were present in the carcinoma mass. Island- or rosette-like cell clusters and stromal amyloid deposition were also present. Immunohistochemical analysis revealed medullary carcinoma, with positive expression of calcitonin and thyroid transcription factor-1. Following surgery, the levels of parathyroid hormone, serum calcitonin and serum CEA were markedly decreased, but still above the standard range. The level of parathyroid hormone was $14.37 \mathrm{pmol} / \mathrm{l}$, the level of serum calcitonin was $416.4 \mathrm{pmol} / \mathrm{l}$ and the level of serum CEA was $13.73 \mathrm{ng} / \mathrm{ml}$.

The wife and daughter of the patient did not possess enlarged neck masses or the manifestations of hypocalcemia. No associated mutations were identified during genetic screening (Fig. 2).

\section{Discussion}

MEN2A is a genetic dominant syndrome resulting from specific RET proto-oncogene mutations, which is characterized by the coexistence of adrenal PHEO, MET and hyperparathyroidism (4) Numerous RET gene mutational sites have been previously reported, $95 \%$ of which are located at 5 coding Cys codons: Codons 609, 611, 618 and 620 of exon 10 and C634R of exon 11. All mutations of codon 634 constitute $73-85 \%$ of RET mutations. The C634Y mutation is the most commonly occurring of the codon 634 mutations, followed by the C634Y mutation in Chinese individuals (5). PHEO mainly occurs in association with codon 634 and 918 mutations, but rarely in association with codon 618 , codon 620 or other mutations (6-8). A previous study reported that the majority of patients with a codon 634 mutation will develop PHEO and MTC during their lifetime (9), as occurred in the case in the present study. A patient with a codon 634 mutation is prone to develop PHEO and MTC at the same time. The diagnosis of PHEO may be determined prior to MTC (12.9-25.1\% of patients), simultaneously with MTC (34.7-38.9\% of patients) or following the diagnosis of MTC (40.2-48.2\% of patients) (10). In addition, 
half of all patients with PHEO may be asymptomatic at diagnosis $(9,10)$. However, the patient and son in the present study were diagnosed with labile hypertension, which is the typical manifestation of PHEO. Patients with typical MTC symptoms present with thyromegaly and pain in the front of the neck prior to the age of 35 years. The symptoms of patients with MTC and C634R mutations are more severe compared with patients only diagnosed with MTC (7). Recently, the American Thyroid Association classified the risk levels of MEN2A, according to all recognized mutations and the aggressive nature of codon 634 (11). The patient in the present study had no evident nodules or neck nodes, or other characteristic manifestations of MTC at the first visit to the hospital. Therefore, the first symptom of the patient was inferred to most probably be PHEO.

Previous studies have reported that the C634R mutation constitutes $54 \%$ of all diseases caused by mutations in the MEN2A family. Additionally, 65\% of all changes to codon 634 and MEN2A patients with a C634R substitution confer an increased risk of developing parathyroid disease compared with other mutations in codon 634 (12). In the present study, the patient was diagnosed with hyperplasia in the parathyroid gland; however, no symptoms or biochemical examination results that are associated with hyperplasia in the parathyroid gland were exhibited by the patient's son, which lead to the hypothesis that there may be a variety of mutations occurring in patients from the same family. In 2001, Mathew et al reported the case of an 18-year-old MEN2A patient with MTC, who succumbed due to multiple metastases. In addition to the C634R mutation, the loss of heterozygosity (retLOH) in exon 4-exon 16 is found in metastasis (13). In 1999, Tessitore et al reported the case of another MEN2A patient who possessed C634R and A640G double mutations (14). Double RET mutations may cause unusual MEN2 manifestations. The 'second hit'-superposition of two variations may be the reason why members of the same family exhibit various phenotypes (15). In the present study, however, the two patients possessed no other mutations. Therefore, in addition to the multiple superimposed effects of mutation, there may be another dysfunction or activation of the proto-oncogene present. The interaction between genetic factors and environmental effects may have lead to the varied clinical manifestations possessed by the patient and his son.

The RET gene mutation has a high specificity and the penetrance of the mutation is almost $100 \%$, which is helpful for the early diagnosis of the disease (16). The current guidelines recommend a DNA analysis of the patient and kin for RET proto-oncogene mutations (6). All individuals with the RET gene mutation require a prophylactic thyroidectomy, and the timing of the surgery is associated with the presence of specific mutations (17). Certain studies consider the sequencing of the RET gene to investigate germline mutations as the standard screening test for MEN2 syndromes (18). The patient in the present study possessed the typical manifestations, biological results and morphological features of MEN2A; however, the patient's son did not exhibit all of these. Therefore, a family history, biological examinations and DNA screening are of high importance in order to ensure the correct diagnosis.

The patient and son were diagnosed with definite MEN2A. The molecular genetic basis of the disease is the 17th base $\mathrm{T} \rightarrow \mathrm{C}$ mutation in the exon $11 \mathrm{rs} 377767437$ sequence. Another proto-oncogene dysfunction or activation in interaction with environmental factor epigenetics may have caused the varying clinical manifestations between father and son.

\section{References}

1. Steiner AL, Goodman AD and Powers SR: Study of a kindred with pheochromocytoma, medullary thyroid carcinoma, hyperparathyroidism and Cushing's disease: Multiple endocrine neoplasia, type 2. Medicine (Baltimore) 47: 371-409, 1968.

2. Farndon JR, Leight GS, Dilley WG, Baylin SB, Smallridge RC, Harrison TS and Wells SA: Familial medullary thyroid carcinoma without associated endocrinopathies: A distinct clinical entity. Br J Surg 73: 278-281, 1986.

3. Ezzat T, Paramesawaran R, Phillips B and Sadler G: MEN 2 syndrome masquerading as MEN 1. Ann R Coll Surg Engl 94: e206-e207, 2012.

4. Muzannara MA, Tawfeeq N, Nasir M, Al Harbi MK, Geldhof $G$ and Dimitriou V: Vaginal delivery in a patient with pheochromocytoma, medullary thyroid cancer, and primary hyperparathyroidism (multiple endocrine neoplasia type 2A, Sipple's syndrome). Saudi J Anaesth 8: 437-439, 2014.

5. Lau GS, Lang BH, Lo CY, Tso A, Garcia-Barcelo MM, Tam PK and Lam KS: Prophylactic thyroidectomy in ethnic Chinese patients with multiple endocrine neoplasia type $2 \mathrm{~A}$ syndrome after the introduction of genetic testing. Hong Kong Med J 15: 326-331, 2009.

6. American Thyroid Association Guidelines Task Force, Kloos RT, Eng C, Evans DB, Francis GL, Gagel RF, Gharib H, Moley JF, Pacini F, Ringel MD, Schlumberger M and Wells SA Jr: Medullary thyroid cancer: Management guidelines of the American Thyroid Association. Thyroid 19: 565-612, 2009.

7. Brandi ML, Gagel RF, Angeli A, Bilezikian JP, Beck-Peccoz P, Bordi C, Conte-Devolx B, Falchetti A, Gheri RG, Libroia A, et al: Guidelines for diagnosis and therapy of MEN type 1 and type 2. J Clin Endocrinol Metab 86: 5658-5671, 2001.

8. Moley JF: The molecular genetics of multiple endocrine neoplasia type 2A and related syndromes. Annu Rev Med 48: 409-420, 1997.

9. Imai T, Uchino S, Okamoto T, Suzuki S, Kosugi S, Kikumori T and Sakurai A; MEN Consortium of Japan: High penetrance of pheochromocytoma in multiple endocrine neoplasia 2 caused by germ line RET codon 634 mutation in Japanese patients. Eur J endocrinol 168: 683-687, 2013.

10. Rowland KJ, Chernock RD and Moley JF: Pheochromocytoma in an 8-year-old patient with multiple endocrine neoplasia type 2A: Implications for screening. J Surg Oncol 108: 203-206, 2013.

11. Krampitz GW and Norton JA: RET gene mutations (genotype and phenotype) of multiple endocrine neoplasia type 2 and familial medullary thyroid carcinoma. Cancer 120: 1920-1931, 2014.

12. Mulligan LM, Kwok JB, Healey CS, Elsdon MJ, Eng C, Gardner E, Love DR, Mole SE, Moore JK, Papi L, et al: Germ-line mutations of the RET proto-oncogene in multiple endocrine neoplasia type 2A. Nature 363: 458-460, 1993.

13. Mathew CG, Smith BA, Thorpe K, Wong Z, Royle NJ, Jeffreys AJ and Ponder BA: Deletion of genes on chromosome 1 in endocrine neoplasia. Nature 328: 524-526, 1987.

14. Tessitore A, Sinisi AA, Pasquali D, Cardone M, Vitale D, Bellastella A and Colantuoni V: A novel case of multiple endocrine neoplasia type $2 \mathrm{~A}$ associated with two de novo mutations of the RET protooncogene. J Clin Endocrinol Metab 84: 3522-3527, 1999.

15. Qi X-P, Ma J-M, Du Z-F, Ying R-B, Fei J, Jin HY, Han JS, Wang JQ, Chen XL, Chen CY, et al: RET germline mutations identified by exome sequencing in a Chinese multiple endocrine neoplasia type $2 \mathrm{~A} /$ familial medullary thyroid carcinoma family. PLoS One 6: e20353, 2011.

16. Gosnell JE, Sywak MS, Sidhu SB, Gough IR, Learoyd DL, Robinson BG and Delbridge LW: New era: Prophylactic surgery for patients with multiple endocrine neoplasia-2a. ANZ J Surg 76: 586-590, 2006

17. Balachandran K, Kamalanathan S, Gopalakrishnan S and Murugananadham K: Multiple endocrine neoplasia 2B: Delayed presentation, rapid diagnosis. BMJ Case Rep 2013: bcr2013009185, 2013.

18. Cui Q, Wang W, Fu Z, Shao X, Zhang Z, Zhang M, Ju X, Wang K, Chen $J$ and Zhou H: Integrated DNA-based/biochemical screening for early diagnosis of multiple endocrine neoplasia type 2A (MEN2A). J Biomed Res 27: 145-150, 2013 